\title{
Estudio por microscopía electrónica y espectroscopía de infra-rojos de capas de SiC obtenidas mediante carburización de obleas de Si
}

\author{
F. M. MORALES', J. RAMÍREZ², C. FERNÁNDEZ 3 , L. BARBADILLO', J PIQUERAS ${ }^{4}$, D. ARAÚJO', S. I. MOLINA', R. GARCÍA \\ 'Dpto. de Ciencia de los Materiales e IM y QI. Universidad de Cádiz, Puerto Real, 11510-(Cádiz). España. \\ ${ }^{2}$ Dpto. de Matemáticas. Universidad de Cádiz, Puerto Real, 11510-(Cádiz). España. \\ ${ }^{3}$ Dpto de Química-Física. Universidad de Cádiz, Puerto Real, 11510-(Cádiz). España. \\ ${ }^{4}$ Laboratorio de Microelectrónica, Facultad de Ciencias, C-XI 100, Universidad Autónoma de Madrid, 28049 Cantoblanco (Madrid). España.
}

\begin{abstract}
Se presentan la fabricación y caracterización de capas delgadas de carburo de silicio $(\mathrm{SiC})$ obtenidas mediante deposición química de vapores por elevación rápida de la temperatura (RTCVD). El proceso de carburización se lleva a cabo utilizando una mezcla de $\mathrm{H}_{2}$ y $\mathrm{C}_{3} \mathrm{H}_{8}$ como único gas precursor de C. Se carburizaron trozos de obleas de Si (001) a presión atmosférica y Si (111) y (001) en condiciones de vacío. Se han utilizado técnicas de microscopía electrónica de barrido (SEM), microscopía electrónica de transmisión convencional (CTEM), de alta resolución (HREM) y de difracción de electrones (TED) así como espectroscopía de infra-rojos por transformada de Fourier en su variante de reflectividad (R-FTIR) para el estudio de la composición y estructura de las capas delgadas obtenidas. Estas capas consisten mayoritariamente en 3C-SiC altamente orientado sobre el sustrato.
\end{abstract}

Palabras clave: Carburización de Si, SiC, RTCVD, TEM, FTIR

\section{Transmission Electron Microscopy study of SiC layers obtained by carbonization of Si wafers}

The fabrication and characterization of thin SiC layers obtained by Rapid Thermal Chemical Vapour Deposition (RTCVD) are reported. These $\mathrm{SiC}$ layers were grown in a simple home-made system for $\mathrm{Si}$ wafers carbonization. The growth process was carried out using a mixture of $\mathrm{H}_{2}$ and $\mathrm{C}_{3} \mathrm{H}_{8}$ as the $\mathrm{C}$ precursor. (001) $\mathrm{Si}$ was carbonized at atmospheric pressure while (111) and (001) Si surfaces were carbonized at vacuum conditions. Scanning electron microscopy (SEM) and transmission electron microscopy in conventional (CTEM), high resolution (HREM), and electron diffraction (TED) modes, as well as reflectivity Fourier transform infra-red spectroscopy (R-FTIR) were the techniques used for the structural and compositional studies of these $\mathrm{SiC}$ layers that mainly consisted in highly oriented 3C-SiC.

Keywords: Si carbonization, SiC, RTCVD, TEM, FTIR

\section{INTRODUCCIÓN}

El $\mathrm{SiC}$ es un material atractivo y versátil con aplicaciones mecánicas por ejemplo en dispositivos micro-electromecánicos (MEMS) [1] o como material abrasivo. Desde el punto de vista electrónico es un semiconductor de banda prohibida ancha con aplicaciones en dispositivos opto y micro-electrónicos que operen en condiciones extremas tales como altas temperaturas, altas frecuencias, altas potencias, radiaciones o ambientes químicamente agresivos [2]. La carburización del silicio permite la consecución de capas de SiC del politipo cúbico ( $3 \mathrm{C}$ o $\beta$ ) de buena calidad mediante deposición química en fase vapor por elevación rápida de la temperatura (RTCVD) [3,4]. Estas estructuras $\mathrm{SiC} / \mathrm{Si}$ son plantillas ideales para el posterior recrecimiento de diamante, de $\mathrm{SiC}$ [5] o de aleaciones cúbicas o hexagonales de tipo III-N como el GaN [6]. La microestructura de los granos y defectos del SiC obtenido podría deteriorar la calidad cristalina del material posteriormente crecido. Para estudiar dicha microestructura se han utilizado técnicas de SEM y TEM. Otra técnica usada para tener un muestreo más rápido es la R-FTIR que permite averiguar las implicaciones ópticas (algunos parámetros ópticos) del $\mathrm{SiC}$ obtenido.

\section{MATERIALES Y MÉTODO}

Las estructuras $\mathrm{SiC} / \mathrm{Si}$ que se estudian fueron obtenidas mediante RTCVD en un sistema de carburización de obleas de Si de fabricación y puesta a punto propias. El diseño del reactor de carburización está basado en el utilizado por Steckl y Li [5] pero se han simplificado partes de la cámara de reacción. De este modo, el flujo de los gases implicados se produce dentro de un tubo de aleación de $\mathrm{Fe}-\mathrm{Cr}-\mathrm{Al}$ para alta temperatura (Incoloy MA956) que pasa por el interior de un horno eléctrico tubular (Carbolite CTF 15/75) de $6 \mathrm{KW}$ de potencia. El horno se regula mediante un controlador electrónico (Eurotherm) y calienta exteriormente al tubo de reacción. El tubo es refrigerado en los extremos de su paso por el horno y en su interior se dispone un dispositivo para trasladar un portamuestras de BN mecanizable cerca del que se ubica un termopar tipo K. Este sistema interno permite el movimiento del portamuestras sin ruptura del vacío mediante unos cilindros magnéticos de teflón y neodimio sinterizado, concéntricos al sistema. Finalmente, un sistema de flujo de gases y de bombeo permite trabajar a diferentes presiones. La fig. 1. muestra una fotografía y un esquema del sistema completo diseñado y construido con el fin de carburizar Si y un esquema de la cámara de reacción. Una descripción más detallada de este equipo puede encontrarse en otras publicaciones [3]. El proceso de carburización para el presente trabajo se lleva a cabo utilizando una mezcla de $\mathrm{H}_{2} \mathrm{y}_{3} \mathrm{H}_{8}$ a presión atmosférica y en condiciones de vacío. Las muestras obtenidas en vacío se carburizaron a $1180^{\circ} \mathrm{C}$ durante 4 a 8 min utilizando una rampa de calentamiento de aproximadamente $5^{\circ} \mathrm{C} / \mathrm{s}$ y se dividen en dos grupos. El primer bloque fue carburizado utilizando una mezcla de flujos de $21 \mathrm{de}_{2} / \mathrm{min}$ con cantidades que oscilaron desde 0,25 hasta $1 \mathrm{ml} \mathrm{de} \mathrm{C}_{3} \mathrm{H}_{8} / \mathrm{min}$. El resto 


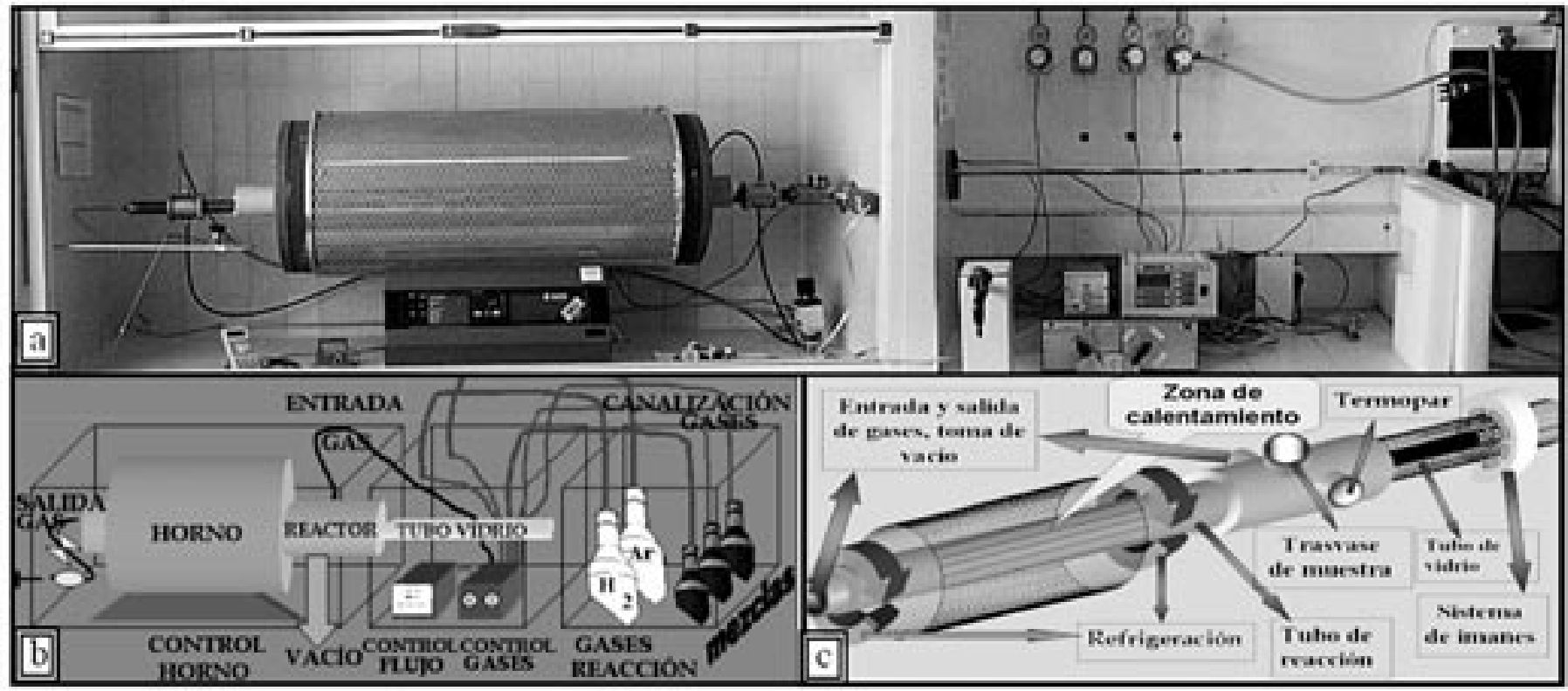

Fig. 1. Fotografía (a) y esquema (b) del sistema de carburización. Esquema del reactor de carburización (c).

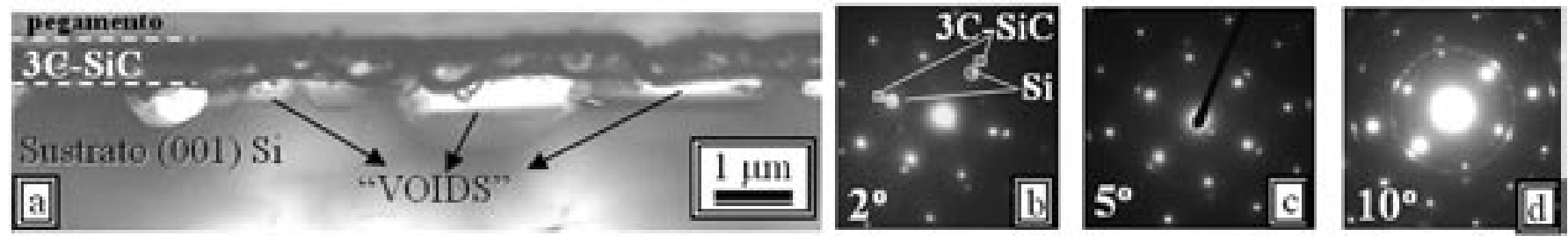

Fig. 2. Imagen de XTEM de la estructura 3C-SiC/(001)Si (a). Diagramas SAED de distintas muestras donde se indica la rotación del arco de difracción de la reflexión de $\mathrm{SiC}$, que aumenta a mayor aporte de $\mathrm{C}(\mathrm{b}-\mathrm{d})$.

de muestras se carburizaron disminuyendo los flujos y variando las proporciones con unos caudales de gas portador de 0,5 y 0,11 de $\mathrm{H}_{2}$ /min. En todos los casos, previamente a la carga dentro del reactor, las muestras de Si fueron desengrasadas en acetona y etanol, más tarde desprendidas de óxido nativo durante 15 min en una disolución de $\mathrm{HF}$ al $5 \%$ y secadas con una pistola de $\mathrm{N}_{2}$. Una vez dentro de la cámara se redujeron en $\mathrm{H}_{2}$ a $1000^{\circ} \mathrm{C}$ para limpiarlas de óxido y tras enfriar se introdujo la mezcla de gases y comenzó la rampa de temperatura.

CTEM, HREM y SAED fueron las técnicas empleadas para el estudio de la micro y nanoestructura cristalina interna de las muestras, mientras las medidas por R-FTIR permiten un estudio desde un punto de vista más macroscópico. Las muestras fueron preparadas para TEM en sección transversal (XTEM) y en visión planar (PVTEM) usando adelgazamiento mecánico y bombardeo de iones $\mathrm{Ar}^{+}$a 4,5 KV en un equipo Gatan Dual Ion Mill. Los estudios de SEM se llevaron a cabo en un microscopio electrónico de barrido JEOL 820 JSM, los de CTEM en un microscopio electrónico de transmisión JEOL JEM-1200EX mientras que la técnica de HREM se desarrolló en un equipo JEOL JEM2000EX/THR. Los espectros de R-FTIR se tomaron con espectrómetros Bruker IFS-66V y Nicolet Impact 410.

\section{RESULTADOS EXPERIMENTALES Y DISCUSIÓN}

La carburización de Si (001) a presión atmosférica produjo capas continuas y planas de $\mathrm{SiC}$ donde el politipo cúbico 3C-SiC fue la fase predominante y donde se observó una alta presencia de policristales hexagonales [3]. En las muestras obtenidas en vacío, en ambas superficies se ha observado una mejora en la calidad estructural del SiC ya que la carburización da origen a capas completamente cúbicas y altamente orientadas sobre el sustrato de Si. Las imágenes XTEM de capas de $\mathrm{SiC}$ obtenidas a altos flujos muestran capas de $\mathrm{SiC}$ de centenas de nanometros de espesor $(400-700 \mathrm{~nm})$ bajo las que se disponen huecos o "voids" típicos de estas estructuras. Estas grandes vacantes son el resultado de la difusión del Si del sustrato hacia el exterior para reaccionar con el C y formar la capa carburizada. En el presente estudio, los "voids" adquieren profundidades del orden del espesor de la capa de $\mathrm{SiC}$ y anchuras que suelen superar $1 \mu \mathrm{m}$. La fig. 2 (a) muestra un ejemplo de estas estructuras. Los diagramas de difracción de electrones de área seleccionada (SAED) correspondientes a estas capas y tomados en eje de zona [011] muestran la total predominancia de las estequiometrías de $\beta$-SiC y Si. El alineamiento de los puntos de difracción correspondientes a estos dos materiales indica, además, la alta orientación existente entre las dos estructuras cuya característica común es la conocida como estructura mosaico, donde cristales de $\mathrm{SiC}$ con una pequeña rotación entre unos y otros se disponen orientados sobre los planos del Si. Esta rotación es identificable en los diagramas de SAED y está relacionada con el arco que se forman en la difracción asociada a reflexiones del 3C-SiC. El arco medido en los SAED de las figuras 2 (b) $2^{\circ}$, (c) $5^{\circ}$ y (d) $10^{\circ}$ aumenta con el aporte de $\mathrm{C}$ en la reacción (fig. 2 (b): $0,4 \mathrm{ml} \mathrm{C}_{3} \mathrm{H}_{8} / \mathrm{min}$; fig. 2 (c): $0,5 \mathrm{ml} \mathrm{C}_{3} \mathrm{H}_{8} / \mathrm{min}$; fig. 2 (d): $0,75 \mathrm{ml} \mathrm{C}_{3} \mathrm{H}_{8} / \mathrm{min}$ ).

Si la carburización es drástica (tiempos de reacción largos o alto aporte de C) se origina una difusión de Si tan acusada que las capas tienden a separarse del sustrato. Esto ocurre porque los voids crecen tanto que casi llegan a unirse y la capa de $\mathrm{SiC}$ queda fijada al $\mathrm{Si}$ mediante pequeñas columnas. Este comportamiento lleva asociado la formación de grietas en las capas de $\mathrm{SiC}$ ya que capas tan delgadas, tensadas y no soportadas tienden a quebrarse como consecuencia del 

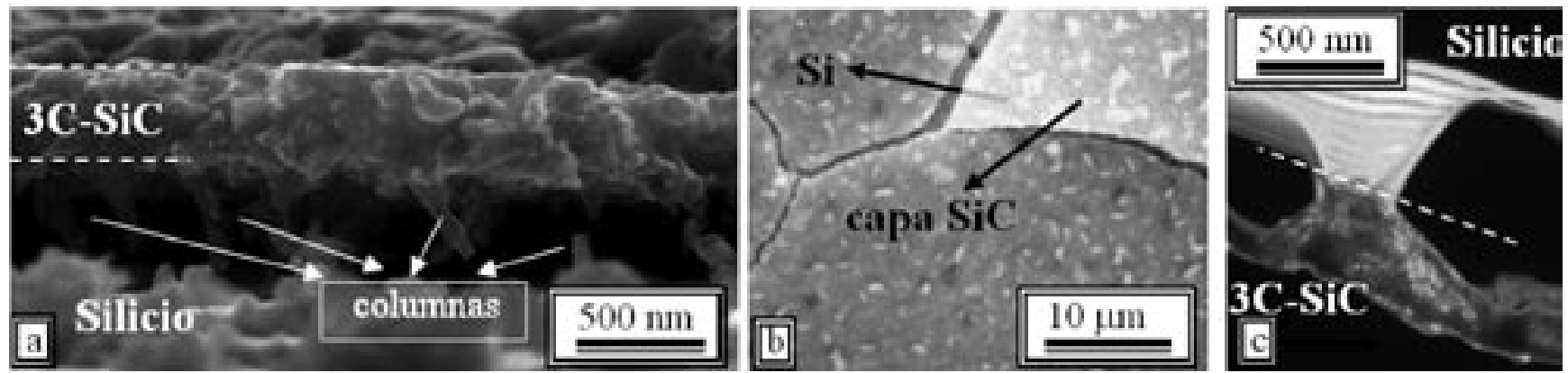

Fig. 3. Imagen del SiC decapado en visión transversal (a) y en plano (b). Imagen DF de la unión SiC-Si (c).

alto desajuste reticular con el $\mathrm{Si}$, cercano al 20\%. Este curioso comportamiento puede ser visualizado en la fig. 3. La fig. 3 (a) muestra una imagen de SEM en sección transversal donde la capa de $\mathrm{SiC}$ se dispone sobre grandes cavidades vacías de material. La fig. 3 (b) muestra una grieta en una capa de $\mathrm{SiC}$ en visión planar. Es posible observar la superficie del $\mathrm{Si}$ debajo del $\mathrm{SiC}$ entre la grieta e incluso a través del $\mathrm{SiC}$ que es relativamente transparente. La fig. 3 (c) muestra una imagen de campo oscuro de una de esas columnas y de la evidente unión entre el SiC y el Si. Otros factores asociados a estas carburizaciones desmesuradas en espesor son la formación de una mayor policristalinidad y porosidad como se muestra en la fig. 4 (a). Los granos de SiC muestran distintos contrastes en PVTEM y la difracción asociada (fig. 4 (b)) exhibe anillos debidos a difracción de policristales con alta desorientación. Las muestras carburizadas a flujos y/o tiempos menores ayudan a averiguar cual es el mecanismo de crecimiento implicado en la formación de las capas ya que el proceso se encuentra en un estado menos avanzado. La fig. 4 (c) es una micrografía de SEM donde varios granos de SiC no unidos están identificados. Un mecanismo de crecimiento tridimensional sin saturación o de tipo Stranski-Krastanov da explicación a lo ocurrido. Para conseguir la mejor monocristalinidad en las muestras, las reacciones deben ser más cortas en tiempo y deben perseguir el seguimiento de un mecanismo de crecimiento cuasi-dimensional con saturación rápida que daría lugar a capas ultra delgadas [7].

Finalmente, el uso de FTIR ha permitido estudiar el comportamiento óptico del $\mathrm{SiC}$ obtenido. El método que introdujeron Hass y Henvis [8] para determinar las constantes ópticas de la reflectividad medida en aleaciones III-N, usando un modelo de oscilador elástico de dispersión simple es aplicado en este caso. Consiste en utilizar las ecuaciones que relacionan la reflectividad $(\mathrm{R})$ de la radiación que incide en una capa de un material absorbente con el índice de refracción complejo. Finalmente, se llega a un sistema de ecuaciones donde el problema se reduce al ajuste de los datos experimentales en función de los parámetros $\omega_{\mathrm{TO}}$ (frecuencia de resonancia asociada a la vibración transversal de los fonones de $\mathrm{SiC}$ a alta longitud de onda), $\omega_{\text {LO }}$ (frecuencia de vibración longitudinal del fonón), $\varepsilon_{0}$ (constante dieléctrica estática o de baja frecuencia), $\varepsilon_{\infty}$ (constante dieléctrica de alta frecuencia) y el factor $\gamma$ (coeficiente de ajuste "damping"). Los cuatro primeros parámetros se relacionan por $\left(\omega_{\mathrm{TO}} / \omega_{\mathrm{LO}}\right)^{2}=\varepsilon_{0} / \varepsilon_{\infty}$. En la fig. 5 se muestran las curvas experimental (marcada con una flecha) y teóricas de ajuste para una muestra $\mathrm{SiC} / \mathrm{Si}$. Con objeto de identificar los parámetros, en el proceso de ajuste se dejan variar libremente partiendo de valores aceptados para el $\mathrm{SiC}$ masivo. De esta forma se ha podido determinar que la presencia de huecos en las intercaras y de cierta saturación de carbono en el $\mathrm{SiC}$, hacen que las capas obtenidas se desvíen de la idealidad que supone una estructura totalmente libre de defectos y que esto afecte a sus propiedades ópticas. Para la fig. 5, los parámetros calculados fueron: $\omega_{\mathrm{TO}}=807,0 \mathrm{~cm}^{-1} ; \omega_{\mathrm{LO}}=999,2 \mathrm{~cm}^{-1} ; \varepsilon_{\infty}=7,5 ; \varepsilon_{0}=11,4$ у $\gamma / \omega_{\mathrm{TO}}=0,015$ partiendo de los valores del cristal ideal $\omega_{\mathrm{TO}}=796,2 \mathrm{~cm}$ ${ }^{1} ; \omega_{\mathrm{LO}}=971,1 \mathrm{~cm}^{-1} ; \varepsilon_{\infty}=6,5 ; \varepsilon_{0}=9,7$ y $\gamma / \omega_{\mathrm{TO}}=0,010$.

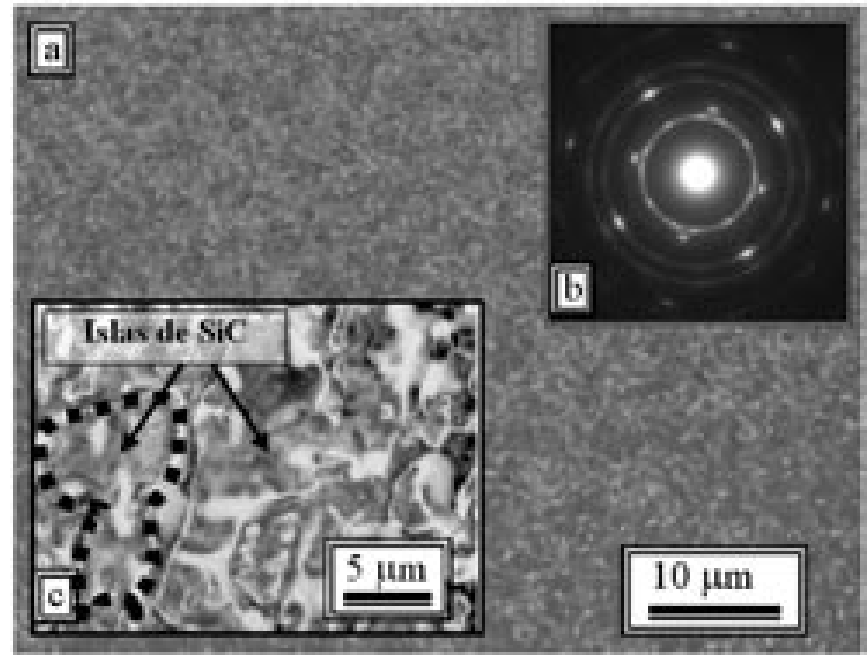

Fig. 4. Imagen PVTEM (a) y SAED (b) del SiC policristalino. Imagen de SEM de islas 3D de $\mathrm{SiC}$ (c).Fig. 5. Espectros R-FTIR experimental (con flecha) y calculados para una muestra 3C-SiC/Si.

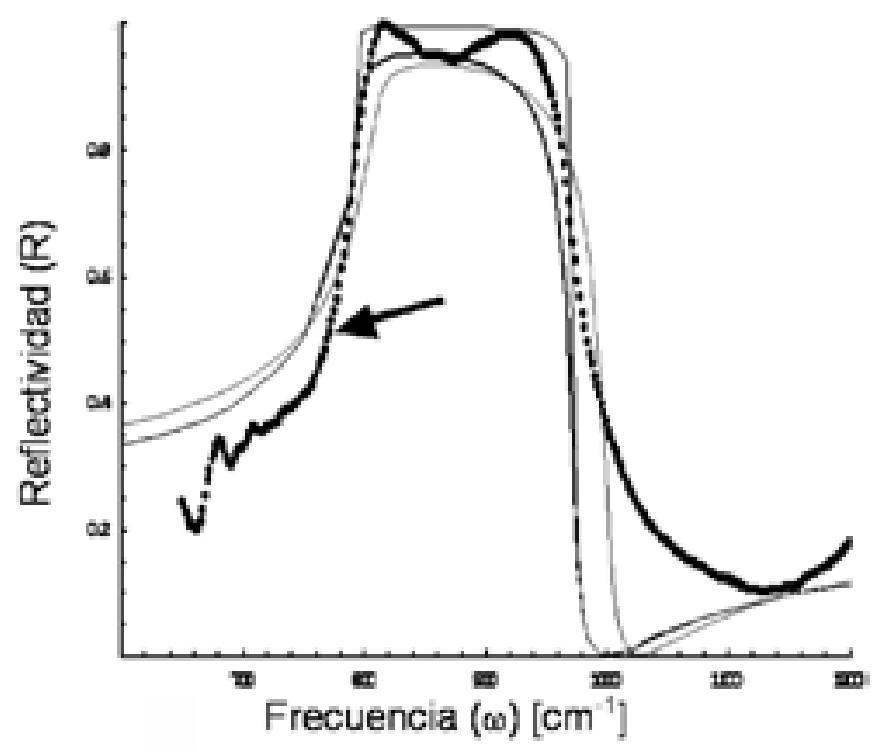

Fig. 5. Espectros R-FTIR experimental (con flecha) y calculados para una muestra $3 \mathrm{C}-\mathrm{SiC} / \mathrm{Si}$. 


\section{CONCLUSIONES}

El estudio de capas 3C-SiC/Si carburizadas a baja presión reflejó un mecanismo de crecimiento tridimensional sin saturación (StranskiKrastanov). Los granos de $\mathrm{SiC}$ dan lugar a una estructura mosaico de $\mathrm{SiC}$ bien orientado sobre el Si que los sustenta. Tiempos de reacción largos o alto aporte de $\mathrm{C}$ provocan la formación de capas de SiC cúbico con poca policristalinidad que tienden a quebrarse y separarse del sustrato.

\section{BIBLIOGRAFÍA}

1. M. Mehregany y C. A. Zorman. "SiC MEMS: opportunities and challenges for applications in harsh environments". Thin Solid Films 355-356, 518-524 (1999).

2. P. G. Neudeck. "SiC Technology," en The VLSI Handbook, The Electrical
Engineering Handbook Series, W.-K. Chen, Ed. Boca Raton, Florida: CRC Press y IEEE Press, pp. 6.1, 2000.

3. S. I. Molina, F. M. Morales y D. Araújo, "SiC thin films obtained by Si carbonization“. Mat. Sci. Eng. B 80, 342-344 (2001).

4. F. M. Morales, S. I. Molina, D. Araújo, V. Cimalla, J. Pezoldt, L. Barbadillo, M. J. Hernández y J. Piqueras. “Transmission Electron Microscopy study of ultra-thin SiC layers obtained by Rapid Thermal Carbonization of Si wafers" phys. stat. sol. (a) 195 [1], 116-121 (2003).

5. A. J. Steckl y J. P Li. "Epitaxial growth of $\beta-\mathrm{SiC}$ on $\mathrm{Si}$ by RTCVD with $\mathrm{C}_{3} \mathrm{H}_{8}$ and $\mathrm{SiH}_{4}^{\prime \prime}$ IEEE Trans. Electron. Dev. 39, 64-74 (1992).

6. F. M. Morales, A. Ponce, S. I. Molina, D. Araújo, R. García, J. Ristic, M.-A. Sánchez-García, E. Calleja, V. Cimalla y J. Pezoldt. "Comparative TEM investigation of MBE and RTCVD conversion of Si into SiC". Mat. Sci. Forum 433-436, 285-289 (2003).

7. V. Cimalla, W. Attenberger, J. Lindner, B. Stritzker y J. Pezoldt. "Structural investigations of the nucleation and growth of $\mathrm{SiC}$ during rapid thermal conversion of (111)Si". Mater. Sci. Forum, 338-342, 285-288 (2000).

8. M. Hass y B. W. Henvis. "Infrared lattice reflection spectra of III-V compound semiconductors". J. Phys. Chem. Solids, 23, 1099-1104 (1962).

Recibido: 1.2 .03

Aceptado: 30.11 .03 